\title{
Modeling System Test Of English As A Foreign Language As A Web-Based Learning Media
}

\author{
M.Nasir ${ }^{1}$, Mahdi $^{2}$ and Hasyimi ${ }^{3}$ \\ \{mhdnasirdaudy@pnl.ac.id\} \\ ${ }^{1}$ Department of Financial Shari'a Banking, Politeknik Negeri Lhokseumawe,Aceh, Indonesia \\ ${ }^{2}$ Department of Electronics, Politeknik Negeri Lhokseumawe,Aceh, Indonesia \\ ${ }^{3}$ Department of Informatics, Politeknik Negeri Lhokseumawe,Aceh, Indonesia
}

\begin{abstract}
This modeling test is equipped by web-based and multimedia system perform to save time and cost in learning TOEFL. The purpose of this research is to design and build the TOEFL system application as a web-based learning media in TOEFLlearning and test. The research specific target is the TOEFL system that can be used as an ICT learning medium online software website for the developing of TOEFL learning. The method used is quantitative method that starts from literature study, interview, observation, data analysis, model and system design, making of the application and system testing. The simulation TOEFL modeling has been simulated using PHP programming languages. The conclusion of this research is a web-based TOEFL simulation as a medium of online software learning in website for the development of learning TOEFL. The random question system will be worked better if the type of questions provided more on the TOEFL simulation. On the simulation, the same participants will not get the same combination of tests on different tests. This TOEFL system capable to runsign inby user-level identification process first, so that the system access is well controlled. This application can only be accessed by users who have made the sign up process.
\end{abstract}

Keywords: Model, TOEFL Learning, Web, Multimedia

\section{Introduction}

One of the English test models used to measure a person's ability whom English is used not as a mother tongue is Test of English as a Foreign Language (TOEFL) [1]. At the firt time, in many countries TOEFL used as a university entrancerequirement (Hutasulut, 2014).

Nowadays, the need for TOEFL test is increasingly around the world, hence proposed of this research is to perform a design of online TOEFL system as a web-based TOEFL learning media totest TOEFL skills. The problems that arise in this research is how to design a web-based TOEFL learning system by making random questions, the system is able to perform online TOEFL assessment based on TOEFL paper-based assessment standard, building a TOEFL database system containing a set of problem models consisting of questions, answers, and discussion of answers, and how the system handles audio listening data. The goal to be achieved is to design and build web-based learning modeling system in the form of TOEFL system application as a web-based learning media in hopes to facilitate 
and ease in testing TOEFL capabilities [2]. This research is useful as a web-based learning media to test the ability of TOEFL. The results of this system service can help the learning process by answering the TOEFL questions as well as getting the discussion of the problem through the web and can help improve the quality of education, especially English. This system is also able to save time and cost in learning TOEFL.

\section{Literature Review}

\subsection{System Definition}

In defining the system there are two groups: one group that emphasizes the procedure and the second group that emphasizes the components or elements. Systems that emphasize the procedure are defined as a network of interconnected procedures gathered together to perform an activity or complete a particular goal. The system is interpreted as a unified element of connectedness. Some elements can be merged into a unit, group or system component with a specific function. These system components can be viewed, considered, or indeed designed to function independently as system modules [3]. The component system defines the system as interconnected and operating parts Together to achieve some goal or purpose.

\subsection{TOEFL form}

Generally, the TOEFL test runs for 3 hours, but for the computer-basedtake time 4.5 hour (Pamela, 2012). TOEFL Tets consist of multiple choice test containing 140 questions divided into 4 sections:

The listening comprehension section consists of 50 questions, Structure and written part of this Expression consists of 40 questions, Vocabulary and Reading Comprehension section consists of 50 questions and Writing Section.

\section{Research Methods}

To achieve the objectives formulated previously, the methodology used in this study is:

\section{Data collection}

Some of the methods to be used in data collection are: library study, interview and observation

\section{Data analysis}

Creating an analysis of data that has been obtained from the data collection process which is then grouped, so that becomes a structured specfication.

\section{System Design}

After the data collected and analyzed then done system design consisting of database design, system modeling diagram context, data flow diagram, flowchart and design of application display.

\section{Application Creation}


This stage is the stage of manufacture and development of applications in accordance with the design system set in the previous stage. TOEFL systemas a learning media is built with PHP and MySQL.

\section{System Testing}

In system testing, all of the overall system specifications will be tested. This trial process is required to ensure that the system that is created is correct, in accordance with the specified characteristics.

\section{Results and Discussion}

\subsection{Results of system simulation}

The result of this TOEFL system simulation begins when member sign in.Member must enter username and password.If the participant has not registered then the participant can not carry out the TOEFL test online in this system.Registration is done online by filling out the sign up form available on the page Home.

\subsection{User Interface page}

The user interface page is an interface display of every function contained in the TOEFL system as a web-based learning media. This can be seen from the results of web view capture.

\subsection{Homes Page}

Inside this page there are several forms of registration sign up form, and sign in form. This page is the only page that can be accessed by web visitors. As for visitors who want to perform the TOEFL test, must first fill in the required data on the sign up form. User interface home page display as shown in Figure 1.

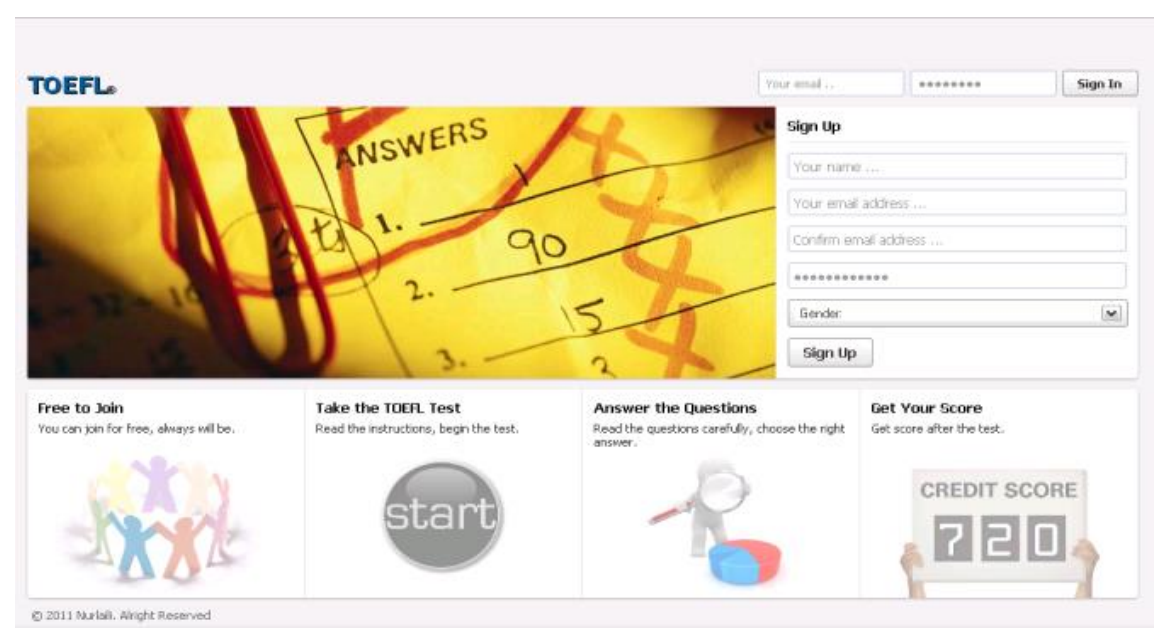

Figure1.Home Page ViewsSign Up

\subsection{Sign In}


The sign in form is used to verify the user in order to access the system, after signing in, the system will check the user that will direct the user to the next page based on the user level by checking the username and password and matching the user data previously stored on Table member if the user level 1 then will be directed to the home page admin whereas if the level 0 then the user is a regular user or member will be directed to the home page member. Display form user interface form sign in shown in Figure2.

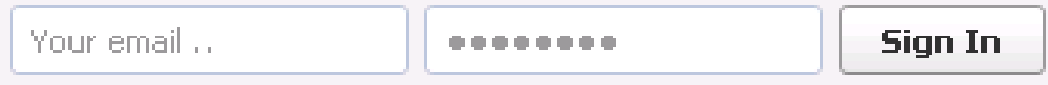

Figure2.Display Form Sign In

\subsection{Contents}

Contents is a means for the admin to input data related to the TOEFL problem.Forms display user interface page contents shown in Figure3.

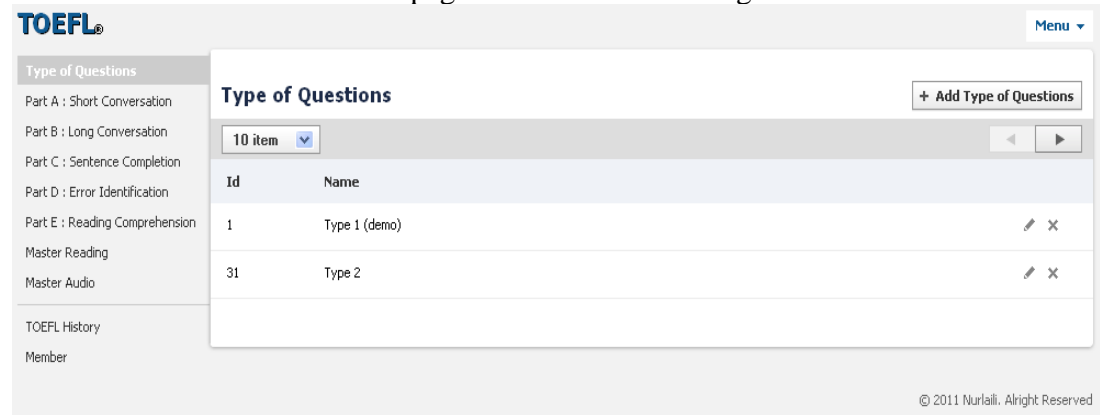

Figure 3.Contents Page Menu View

In the input process of TOEFL problem, the first thing the admin is doing is input data into the menu type of questions. Admin just input the name of the desired type of problem then the systems stores the name of the type of question into the table type and fill in the id_type field automatically. Each additional name of the type of problem then the id_type value will increase 1 because setting auto increment. User interface menu type of question is shown in figure 4.

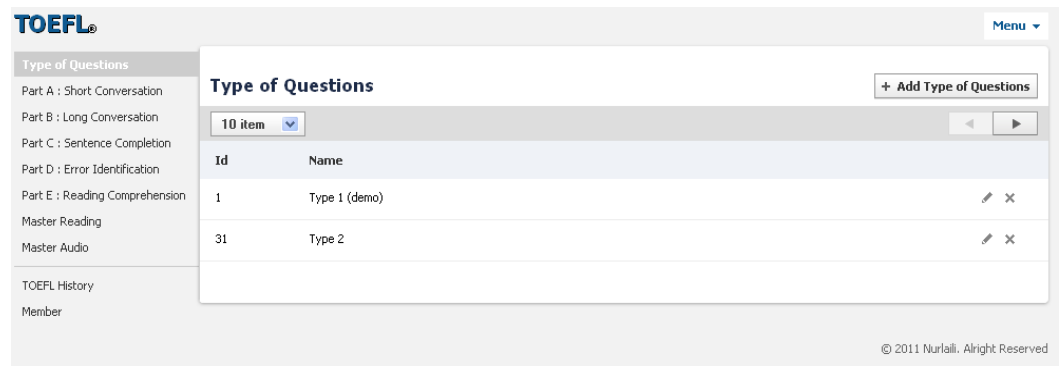

Figure 4. Display Menu Type of Questions

Menu part a: short conversation, is the menu used by admin to input TOEFL listening section short conversation problem such as question, answer choice, answer key, discussion, 
and audio listening short conversation data. The table part a is the table used to store the listening short conversation problem, the table for storing this problem is grouped in order to facilitate the random process of the problem.

In the input form looks some data that can input like type that can be used to choose the name of the type of questions that have been entered earlier, so at this stage admin only choose the type of question he wants. Next audio, used to select the audio file short conversation that has been input to the master audio that will be discussed in the next section. Question is used to insert problem, a, b, c, d are used to input answer option, answer to enter answer key, and last explanation to enter problem discussion.

In addition to the input on this menu there is also the edit function, and delete it by clicking the button with the pencil icon to edit, and the button with the crossbar icon to delete the data part a short conversation. So the MySQL syntax used to perform the functions contained in this menu is insert, update and delete to the data contained in the table part a. This is in accordance with the design shown earlier in the DFD level 1 . The display of the user interface menu of the part a short conversation menu is shown in Figure 5.

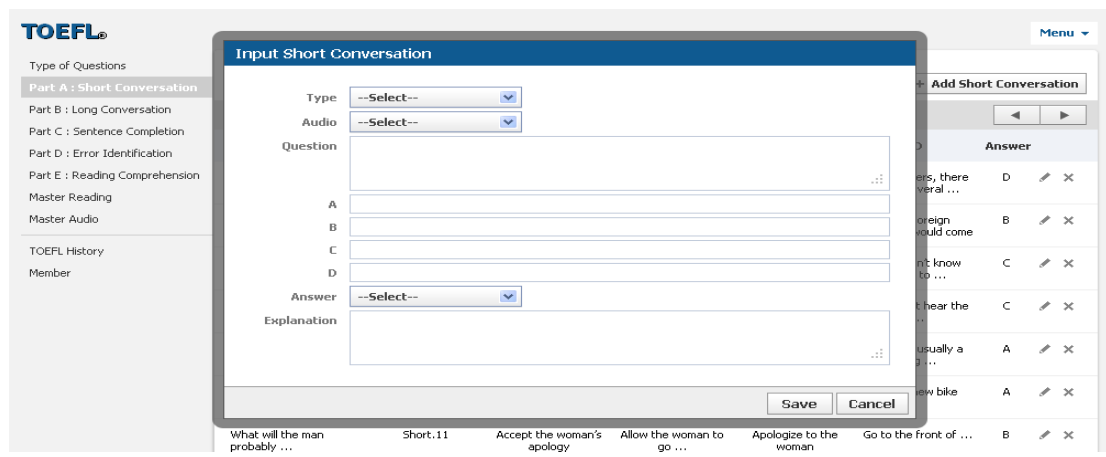

Figure 5. Display Menu Part a: Short Conversation

Menu part b: long conversation, is the menu used by admin to input TOEFL listening section long conversation in the form of questions, answer options, answer key, discussion, and audio listening short conversation data. For input about listening section long conversation is used sintak insert into table part $\mathrm{b}$. The table part $\mathrm{b}$ is the table used to store the listening long conversation problem.

In the form input part $\mathrm{b}$ long conversation visible some data that can input like type that can be used to select the name of the type of questions that have been entered earlier, so at this stage the admin only live Choose the type of question he wants. Next audio, used to select the audio file short conversation that has been inputted to the master audio that will be discussed in the next section. Question is used to insert problem, a, b, c, d are used to input answer option, answer to enter answer key, and last explanation to enter problem discussion. In addition to the input on this menu there is also the edit function, and delete it by clicking the button with the pencil icon to edit, and the button with the crossbar icon to delete the data part b long conversation. So the MySQL syntax used to perform the functions contained in this menu is insert, update and delete data contained data contained in the table part $b$. This is in accordance with the design shown earlier in the DFD level 1. Display the user interface of the menu menu part $\mathrm{b}$ long conversation shown in Figure 6. 


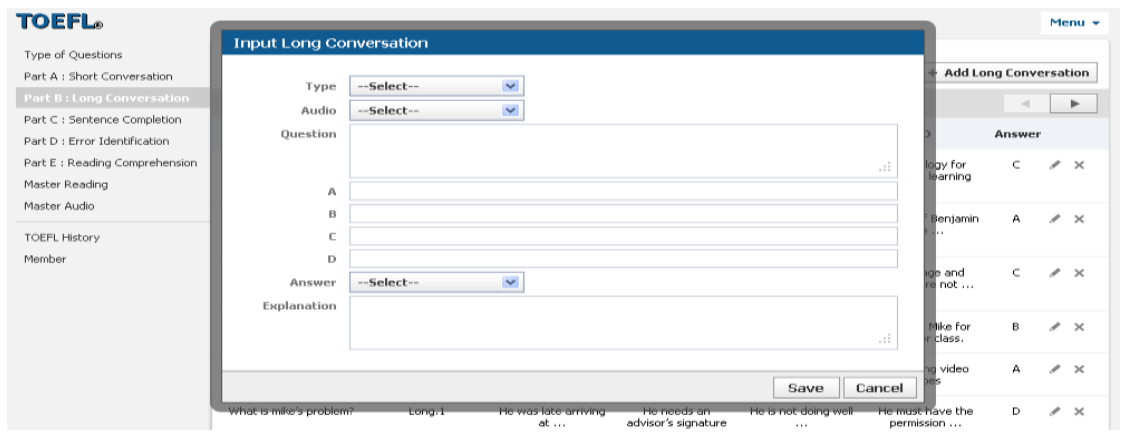

Figure 6. Display Menu Part b: Long Conversation

Menu part c: sentence completion, is menu used by admin to input TOEFL question structure and written expression sentence completion in the form of question, answer choice, answer key, and discussion. For input about structure and written expression sentence completion is used sintak insert into table part $\mathrm{c}$. Table part $\mathrm{c}$ is the table used to store sentence completion only.

In the input menu part $\mathrm{c}$ sentence completion looks some data that can input like type that can be used to select the name of the type of questions that have been entered earlier, so at this stage admin only choose the type of question he wants. Question is used to insert problem, $\mathrm{a}, \mathrm{b}, \mathrm{c}, \mathrm{d}$ are used to input answer option, answer to enter answer key, and last explanation to enter problem discussion. In addition to the input on this menu there is also an edit function, and delete it by clicking the button with the pencil icon to edit, and the button with the crossbar icon to delete the data part c sentence completion. So the MySQL syntax used to perform the functions contained in this menu is inserting, update and delete to the data contained in the table part c. This is in accordance with the design shown on the DFD level 1. Display the user interface interface of the menu part c sentence completion shown in Figure 7.

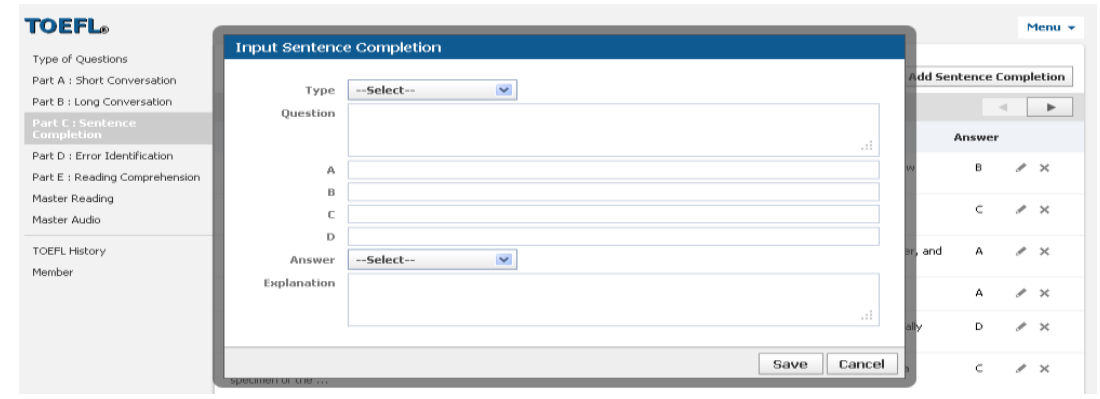

Figure 7. Display Menu Part c: Sentence Completion

Menu part d: error identifications, is menu used by admin to input TOEFL question structure and written expression error identifcation in the form of question, answer choice, answer key, and discussion. For input about structure and written expression error identifcation is used sintak insert into table part $\mathrm{d}$. The table part $\mathrm{d}$ is the table used to store the error identification problem only.

In the form input menu part $\mathrm{d}$ error identification seen some data that can input like type that can be used to choose the name of the type of questions that have been entered earlier, so 
at this stage admin only choose the type of question he wants. Quesion used to enter the problem, a, b, c, d are used to include answer options, answer to enter answer key, and last explanation to enter discussion of problem. In addition to the input on this menu there is also the edit function, and delete it by clicking the button with the pencil icon to edit, and the button with the crossbar icon to delete the data part d error identification. So the MySQL syntax used to perform the functions contained in this menu is inserting, update and delete to the data contained in the table part $d$. This is in accordance with the design shown in the DFD level 1. Display the user interface menu menu part d error identificaton shown in Figure 8.

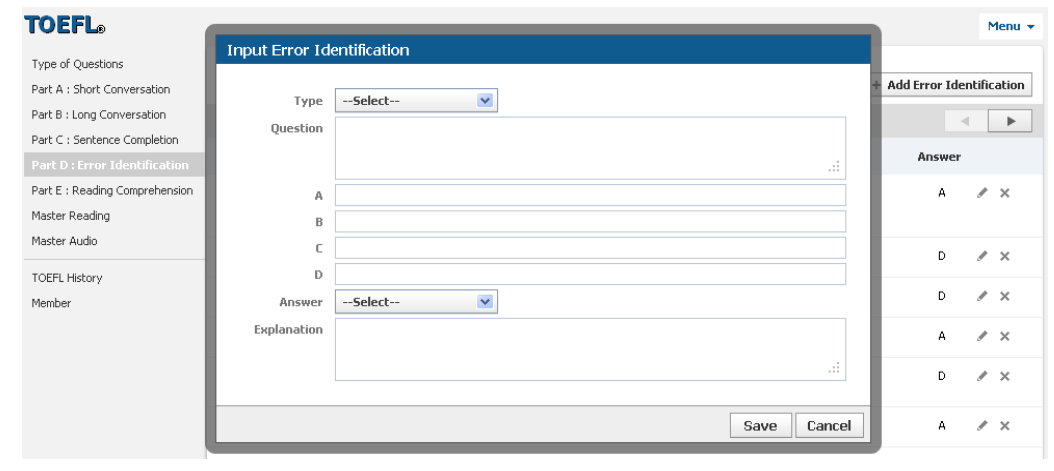

Figure 8. Display Menu Part d: Error Identification

Menu part e: reading comprehension is the menu used by admin to input TOEFL reading comprehension questions in the form of questions, answer choices, answer key, discussion, and reading. For input reading comprehension question is used sintak insert into table part e.

Table part $\mathrm{e}$ is the table used to store reading comprehension only.

In the form input menu part e reading comprehension looks some data that can input like type that can be used to choose the name of the type of questions that have been entered earlier, so at this stage admin only choose the type of question he wants. Reading is used to select the source text that has been inputted in the input process of the master reading.Question used to insert the problem, a, b, c, d are used to enter answer options, answer to enter answer key, and the last explanation to enter the discussion of questions. In addition to the input on this menu there is also the edit function, and delete it by clicking the button with the pencil icon to edit, and the button with the crossbar icon to delete the data part e reading comprehension. So the MySQL syntax used to perform the functions contained in this menu is insert, update and delete to the data contained in table part e.This is in accordance with the design shown in DFD level 1.The user interface form of the reading part reading menu is shown in Figure 9. 


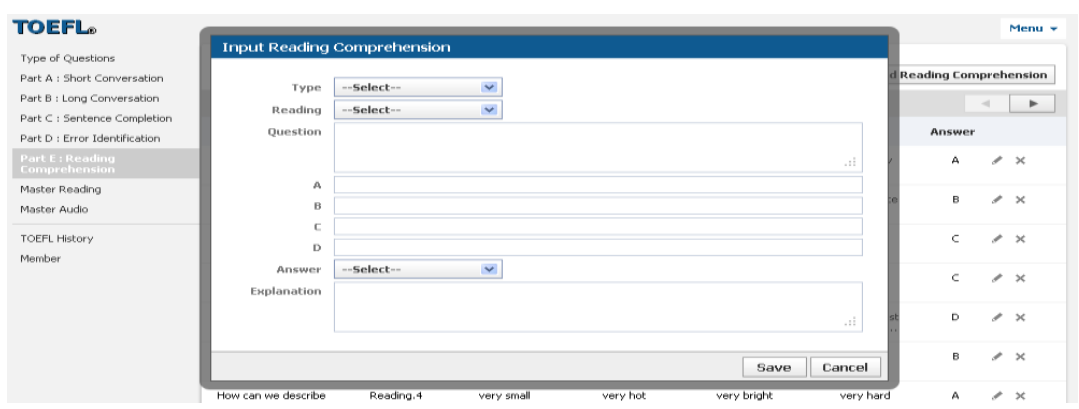

Figure 9. Display Menu Part e: Reading Comprehension

Master reading menu, used for readingtext data needs to be used for TOEFL reading comprehension. On the input menu of the master reading menu there are 3 data types used to select the desired type of title, title to give the title of the text, and reading the contents Is a text that will be used to answer questions about the reading. The table used to store data on this menu is a table of reading that is also related to table part e. Functions contained in this menu is the same as the previous menu that is, insert, update, and delete to the data contained in the master reading table. This is in accordance with the design shown in the DFD level 1. Display menu master reading shown in Figure 10.

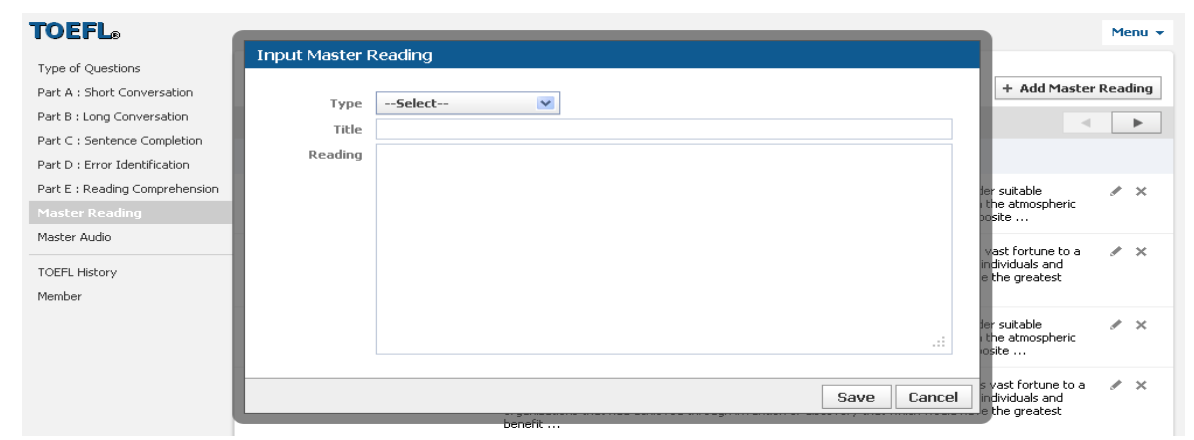

Figure 10. Display Menu Master Reading

The next menu is the audio master menu that is used for the needs of audio data input listening and used for TOEFL listening section. The data after inputted or manipulated will be stored into the audio table that also relate to the table part a and part $\mathrm{b}$. This corresponds to the relation shown in the table relation discussed earlier in the design chapter. The process contained in this menu edit, delete, and input. In addition audio files that are ready to be uploaded into the system can be played by first installing plug in or additional tool used to play audio files on the web iequicktime player. The display form of the audio master menu is shown in figure 11. 


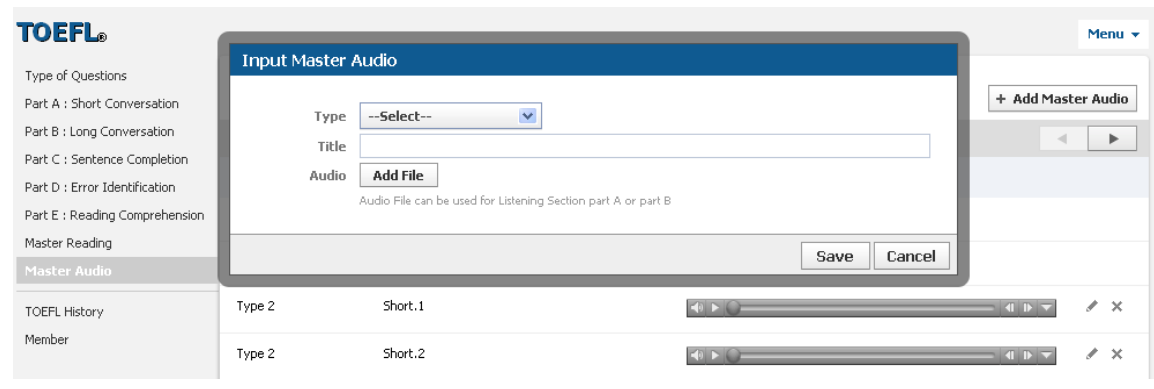

Figure 11. Display Master Audio Menu

TOEFL history menu, admin is used to view the results of score score member, and member log. When member sign in and successfully perform the TOEFL test, the details of time description data, the type of problem, score listening, structure, reading, and TOEFL score stored by the system Into the history table and data that has been stored is called for the needs of this menu. So with this menu admin can see traces of member activity in the system, and admin can monitor a number of members who go into the system and do the TOEFL test.Sintak used for Process function from the TOEFL history menu is select $*$ from history where userid. The display form of the audio master menu is shown in figure 12 .

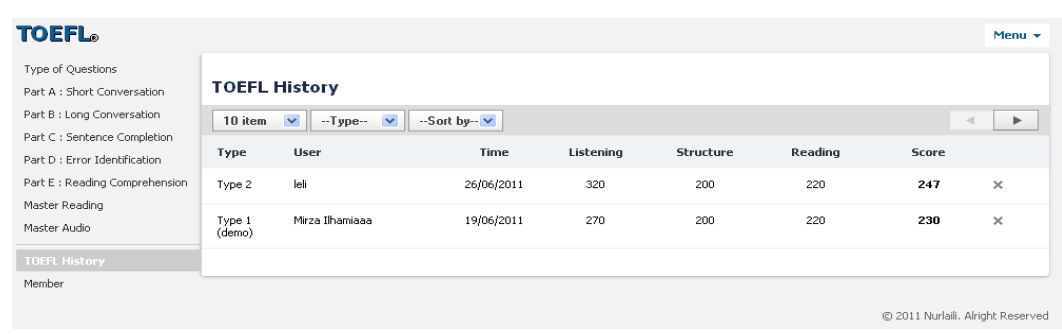

Figure 12. TOEFL Menu display history

Member menu is used by the admin to see the identity data of members who have registered into the system. The user interface display of the member menu is shown in Figure 13.

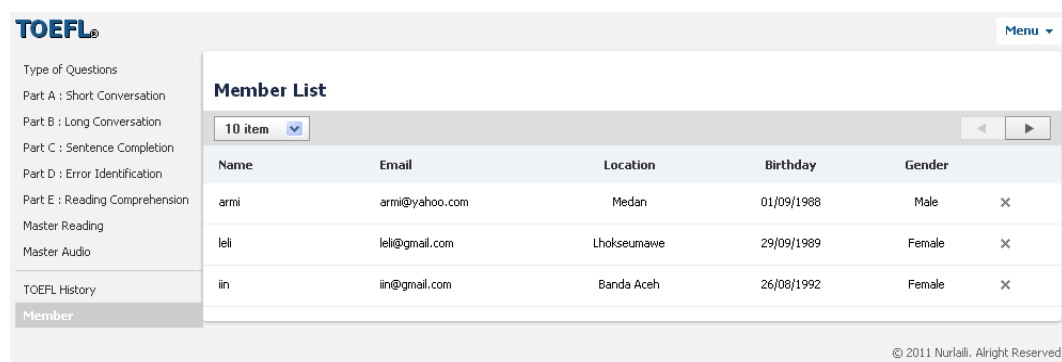

Figure 13. Display Member Menu 


\subsection{Member Home Page}

The home page of the member or the page that first appeared after the member successfully signed in. It is shown in Figure 14

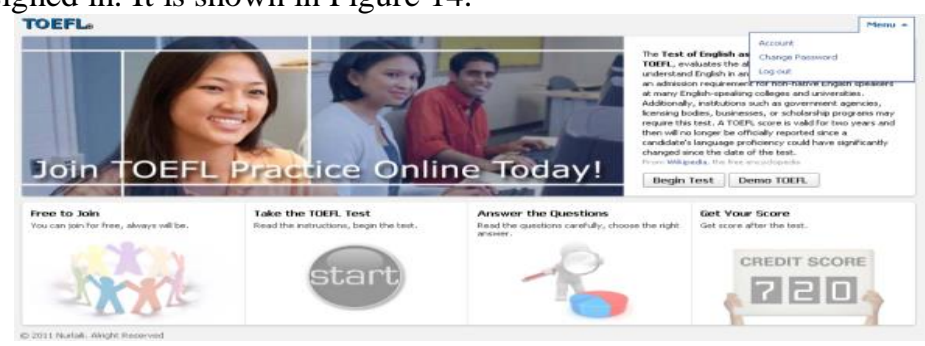

Figure 14.Views of Member Home Page

\subsection{Begin Test}

Begin test menu located on the home page admin and member, is a facility to perform TOEFL test without discussion. Each user can only perform one TOEFL test for each one of the questions provided for the begintest process. This is in accordance with the flowchart design of the begin test that has been described in the previous chapter. Before heading to the page matter, will pop up welcome as follows. Inside is the start button TOEFL test that will lead to the next page, or cancel button to cancel it. Display user interface pop up welcome begin test shown in figure 15 .

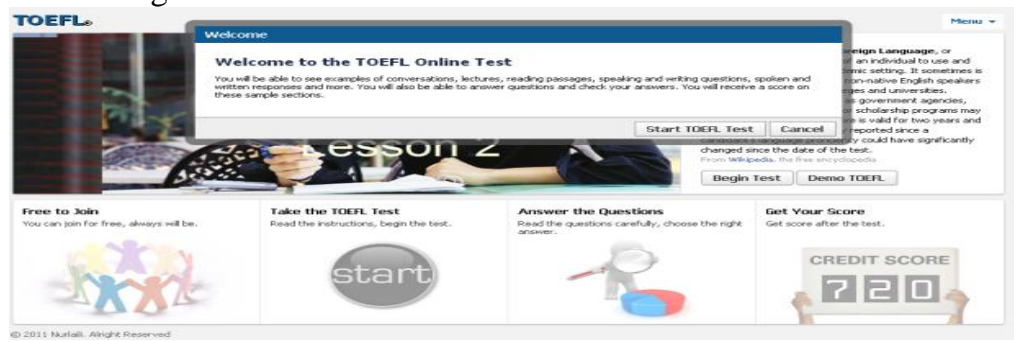

Figure 15.Pop up Display Welcome Begin Test

Then will be displayed pop up section 1 listening comprehension. Pop up contains instructions to perform TOEFL test listening list Display user interface menu pop up section 1 listening comprehension shown in Figure 16.

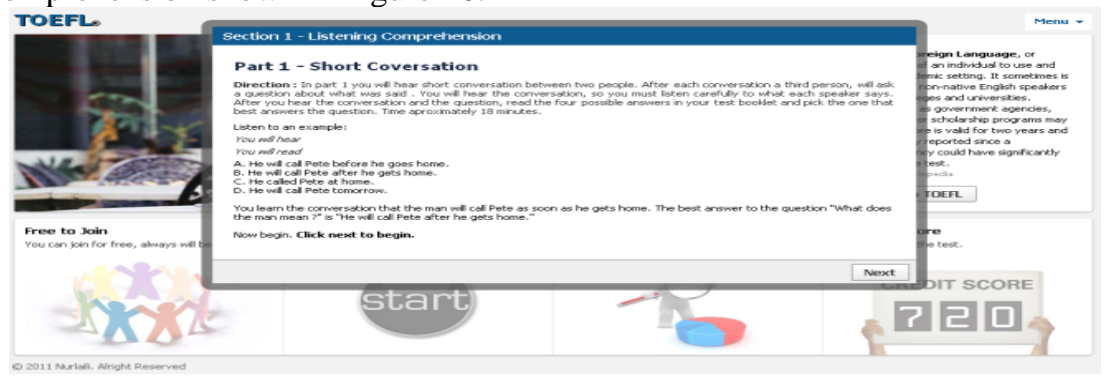

Figure 16.Pop Up Section 1 Listening Comprehension display 
After clicking the next button then the listening for short conversation will appear on the web page along with the choice of answers that will store the member's reply with the condition member must click on one of the radio buttons that indicate the answer that is considered correct by member.The user interface display of listening short conversation is shown in Figure 17.

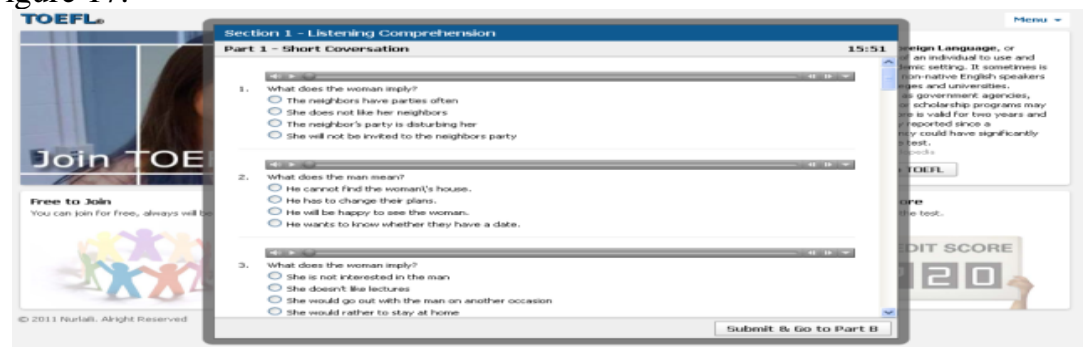

Figure 17.Listening Problem Short Conversation Problem

Furthermore, after the member answers all the short listening listening questions, the system will store the answer into the database table answer. The system will also process the answer correctly or wrongly by checking the answer key that is located in the answer field in the table part a, if the member's correct answer will be given a value of 1 and if the wrong member's answer will be given a value of 0 . Display user interface pop up listening long conversation shown in figure 18 .

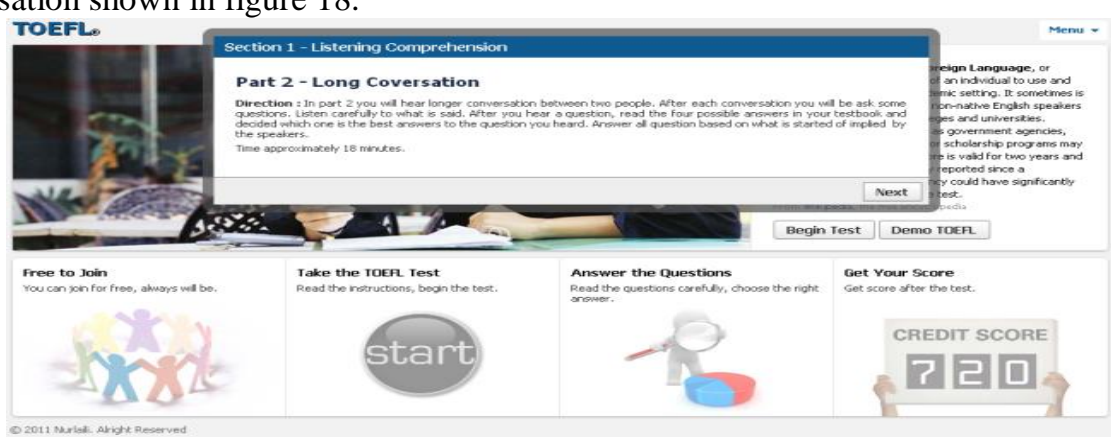

Figure 18.Display of Long Conversation Listening pop up

After the member answers all the listening long conversation questions, the system will store the answer into the database table answer. The system will also process the answer is correct or wrong by checking the answer key that is located in the answer field in the table part $\mathrm{b}$ as shown in Figure 19

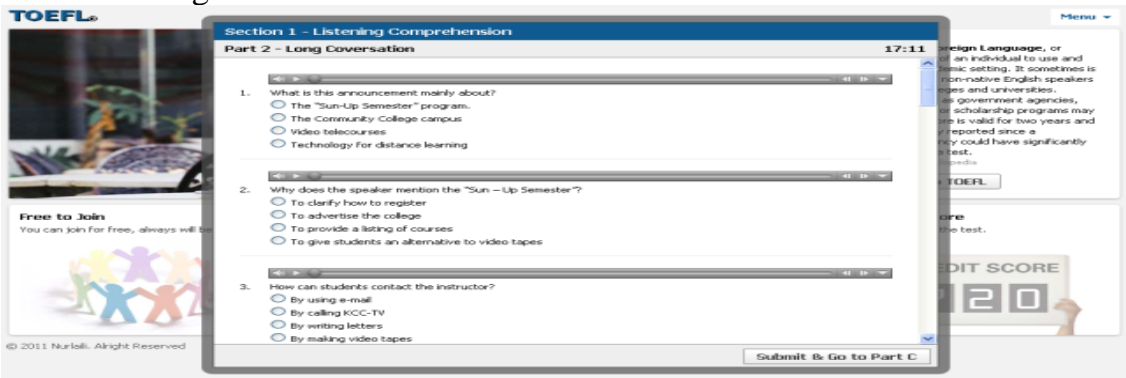

Figure 19.Long Listening Problem List Conversation 
After answering the entire question, clicking on the submit button will pop up a pop up that gives instructions for working on the next question, namely section 2 structure and written expression which amounts to 40. The next button is clicked back then the question will be about structure and written expression sentence completion. The user interface display of the structure and written expression sentence completion is shown in Figure 18.

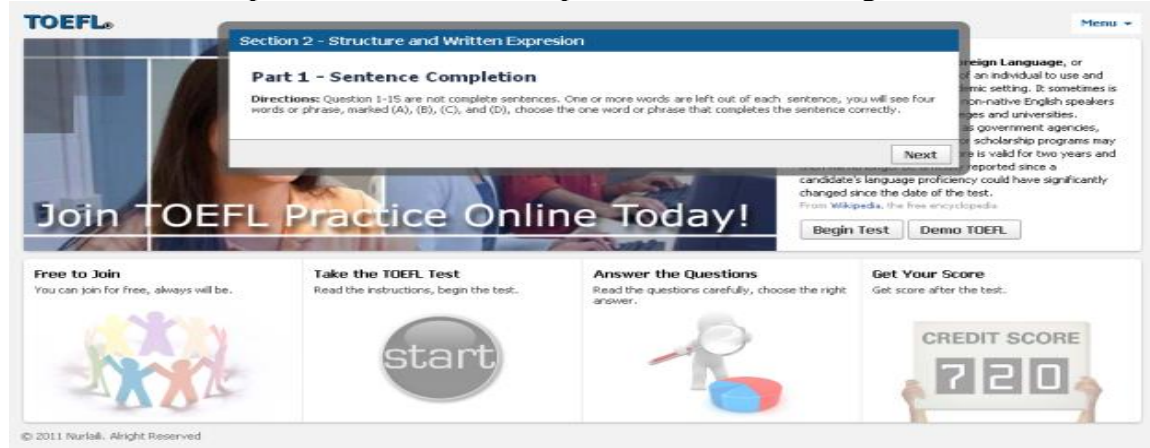

Figure 18.Pop up Structure Written Expression Sentence Completion

After the member answers all the question structure and written expression sentence completion, the system will store the answer into the database table answer. The system will also process the answer is true or wrong by checking the answer key that is located in the answer field in the table part $\mathrm{c}$ as shown in Figure 19.

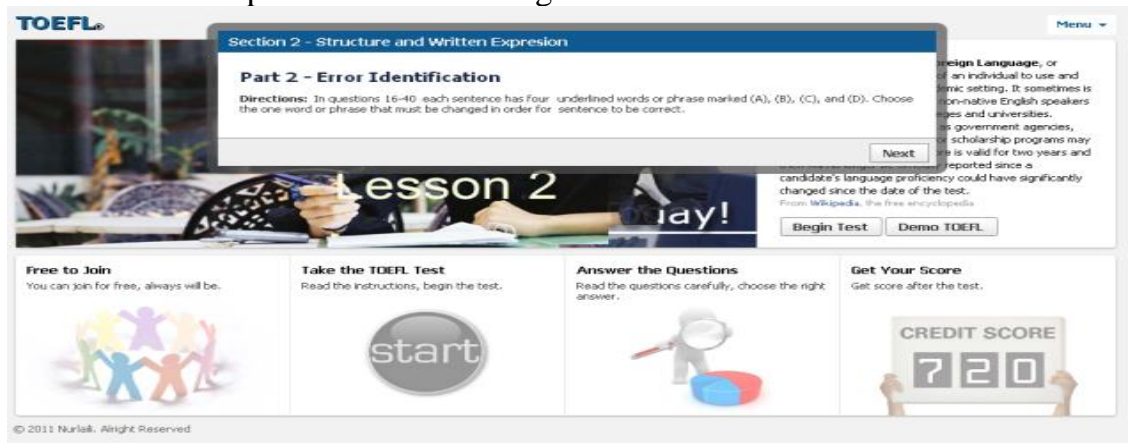

Figure 19.Pop Up Display Structure Written Expression Error Identification

The next button is clicked again and a question about structure and written expression error identification will appear as shown in Figure 20.

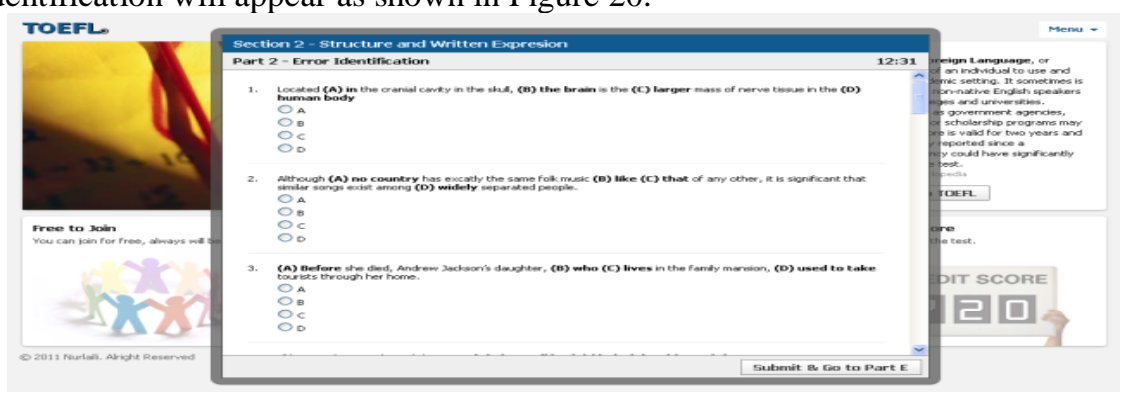

Figure 20.Problem Display Structure Written Expression Error Identification 
After the next button is clicked, it will appear the reading comprehension as shown in Figure 21.

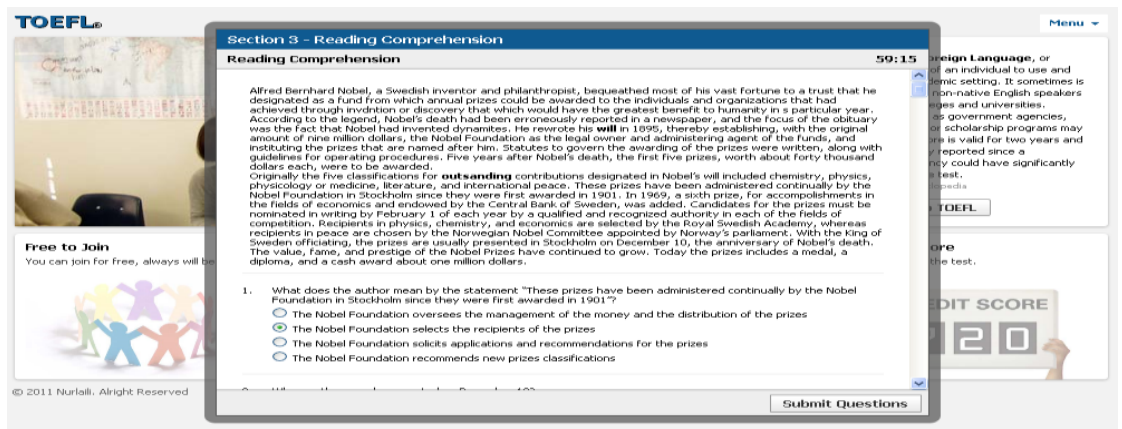

Figure 21.Reading Problem Reading Comprehension

After the member answered all questions reading comprehension, the system will store the answer into the database table answer.System will also process the answer is true or wrong by checking the answer key that is located in the answer field in the table part e. The TOEFL score derived from the calculation is done by summing the total correct answers from each part stored in the answer table and then grouped into 3 sections, then the value of each section is adjusted to the num_correct data is found in the score table to produce a TOEFL score based on PBT standards. TOEFL score for begin test is stored into the history table, and will be called when members access the account menu that is history to see the details of the report score that has been done. The TOEFL user interface display is shown in Figure 22.

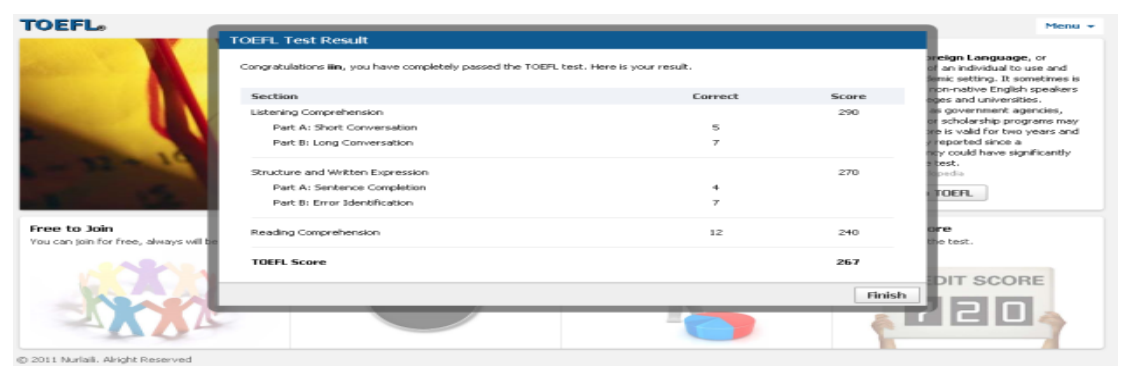

Figure 22.Display of TOEFL Results

\section{Conclusion}

This TOEFL system is able to run sign infor user-level identification process at the beginning so that the system access is well controlled. This application can only be accessed by users who have done the process of signing up. Random system question will be worked better when the type of questions provided more in variant and items of the test. The userwill not get the same combination of tests on different tests for the same participants. The TOEFL system as a learning medium is an online software in the form of a website create for the development of TOEFL learning and test. 


\section{References}

[1] Free English Test diaksespada 1 Februari 2017 dari http://www.english-test.net

[2] Hutasulu, Mirah Diani.2014. Best Strategy of TOEFL. Jakarta: Prima

[3] Jogiyanto HM. 2005. Analisis dan Desain Sistem Informasi. Yogyakarta: Andi.

[4] Sharpe, Pamela J. 2007. TOEFL Ibt, $12^{\text {th }}$ edition. Barron's Educational, Inc

[5] Sharpe, Pamela J. 2002. How to Prepare for the TOEFL. 10 ${ }^{\text {th }}$ edition. Barron's Educational, Inc

[6] Sofwan, Akhmad.2010.Belajar PHP dengan Framework Code Igniter. Jakarta: IKC.

[7] TOEFL iBT® Research.2011.Insight -TOEFL® Program History.NewJersey:ETS. 